\title{
Characterization of an Opioid Peptide-Containing Protein and of Bovine $\alpha$-Lactalbumin by Electrospray Ionization and Liquid Secondary Ion Mass Spectrometry
}

\author{
Lin Yan*, Jih-Lie Tseng, Genevieve H. Fridland, and \\ Dominic M. Desiderio ${ }^{\dagger}$
}

The Charles B. Stout Neuroscience Mass Spectrometry Laboratory, University of Tennessee, Memphis, Memphis, Tennessee, USA

Mass spectrometry methods have been used to characterize two proteins: an opioid peptidecontaining protein extracted from bovine pituitary, and bovine a-lactalbumin (BAL). A protein that contains $\beta$-endorphin was found in bovine pituitary, and that protein was characterized with electrospray ionization mass spectrometry (ESIMS), gel permeation chromatography, reversed-phase high performance liquid chromatography (RP-IPLC), radioimmunoassay, trypsinolysis, and liquid secondary ion mass spectrometry (LSIMS).

BAL is a protein that was used as a model to develop analytical methods to study opioid peptide-containing proteins. Commercial BAL was purified by RP-HPLC, and its molecular weight (M.W.) was determined by ESIMS. The shift in mass observed following dithiothreitol (DTT) reduction estimated the number of disulfide bonds.

For all of the data obtained for BAL with or without RP-HPLC separation, ESIMS determined the M.W. of the peptides produced by trypsin treatment of BAL, and LSIMS selected a precursor ion, the protonated molecule ion $[\mathrm{M}+\mathrm{H}]^{+}$, of a tryptic peptide, which was analyzed by tandem mass spectrometry. Following DTT reduction, ESIMS and LSIMS detected each peptide that contained disulfide bonds in that mixture of tryptic peptides. (J Am Suc Mass Spectrom 1994, 5, 377-386)

$\mathrm{T}$ The qualitative and quantitative analysis of neuropeptides in biologic tissues and fluids is a main research activity for this laboratory [1-13], especially the endogenous opioid and tachykinin neuropeptidergic systems in human pituitary tissue. Mass spectrometry methods have been developed and used to optimize the molecular specificity of those qualitative and quantitative analytical measurements. For example, tandem mass spectrometry (MS/MS) in the multiple reaction monitoring (MRM) mode has been used to quantify endogenous methionine enkephalin (ME) $[5,11]$ and $\beta$-endorphin (BE) [9] in human postmortem control and postsurgical tumor pituitaries $[6$, 7]. We have also shown for the first time in the human trigeminal system, the presence of opioid peptide-containing proteins that lead to ME [14]. Recently, electrospray ionization (ESI) has facilitated the analysis of

\footnotetext{
- Also at the Center for Excellence in Neuroscience, Univcrity of Terunessee, Memphis.

Also at the Deparments of Neurology and Biochemistry and the Center of Excellence in Neuroscience, University of Tennessee, Memphis.

Address reprint requests to Professor Dominic M. Desiderio, De partment of Neurology, University of Termessee, Memphis, 800 Madison Avenue, Memphis, TN 38163 USA.
}

those opioid peptide-containing proteins [15], and will also be used to clarify neuropeptidergic systems in the pituitary.

An opioid neuropeptide derives metabolically in the cell body of a neuron from a protein that contains approximately 260 amino acids [16]. For example, ME and $\mathrm{BE}$ are produced from two different proteins, preproenkephalin A (263 amino acids) and proopiome lanocortin (POMC) (265 amino acids), respectively, through one or more intermediate-sized opioid peptide-containing proteins ("intermediate-sized" means fewer than the $\sim 260$ amino acids in a precursor, but more than the 5 or 31 amino acids in ME or $B E$, respectively). The amino acid sequence of preproenkephalin A and of POMC was deduced by the use of recombinant DNA techniques [16]. However. the number and the characterization of intermediatesized opioid peptide-containing proteins remain uncertain for the steps involved in the metabolism from preproenkephalin A to ME and from POMC to BE.

During axonal transport, proenkephalin $A$ and POMC are metabolized through one or more intermediate-sized opioid peptide-containing proteins to produce the neuropeptide, which is packaged in vesicles. 
For ESIMS, the source accelerating voltage was 4 $\mathrm{kV}$, and the ESI probe tip-to-counter electrode voltage was $3 \mathrm{kV}$. The samples were dissolved in a solvent of $\mathrm{CH}_{3} \mathrm{OH}: \mathrm{H}_{2} \mathrm{O}\left(1: 1\right.$, v:v) containing $1 \% \mathrm{CH}_{3} \mathrm{COOH}_{\text {; the }}$ BAL concentration was $\sim 25 \mathrm{pmol} / \mu \mathrm{l}$. An aliquot (20 $\mu \mathrm{l})$ of the sample solution was introduced into the ESI source at a flow-rate of $3 \mu \mathrm{l} / \mathrm{min}$ with a syringe pump (Harvard Model 22, Woburn, MA).

For an ESIMS scan of a protein, typical MS-1 instrument operating parameters were: mass resolution $=$ $\sim 200$; mass range $=100-2500$; scan-rate $=5 \mathrm{~s} / \mathrm{de}-$ cade; data acquisition time $=10 \mathrm{~min}$; number of scans accumulated $=\sim 100$. Mass calibration was performed with the $[\mathrm{M}+2 \mathrm{H}]^{2+}$ ion of Gramicidin $\mathrm{S}$ at $\mathrm{m} / \mathrm{z}$ 571.4. All data were smoothed and transformed using the VG software.

For LSIMS, $\mathrm{Cs}^{+}$ions with an energy of $35 \mathrm{keV}$ were used. The source accelerating voltage was $8 \mathrm{kV}$. Mass resolution was $\sim 1200$, and glycerol was used as the matrix. The spectra were obtained at a scan-rate of 5 $\mathrm{s}$ /decade, and mass calibration was performed with CsI.

The average mass value was used for the ESIMS data for the opioid peptide-containing protein and for intact BAL, and monoisotopic mass values were used for all LSIMS and all multiply charged ESI ions.

\section{MS / MS}

For MS/MS, LSIMS was used for sample ionization, MS-1 selected the precursor ion, and MS-2 collected the product ion spectrum. MS-2 was operated at 5 $\mathrm{s} / \mathrm{scan}$, and mass resolution was one (at $\mathrm{m} / z$ 500). Helium was used as the collision gas, and the helium pressure within the collision cell was adjusted to reduce the precursor ion current by $\sim 50 \%$.

\section{Results and Discussion}

\section{Bovine Pituitary Opioid Peptide-Containing Protein}

GP and RP-HPLC separation of a bovine pituitary homogenate produced fractions that contained ME-li after treatment with trypsin and $\mathrm{CNBr}$, and those fractions were analyzed by ESIMS to determine the M.W. of the protein.

ESIMS analysis of one of those fractions showed the presence of three major components, with M.W. values of 6550, 8440, and $9855 \mathrm{Da}$, respectively. Figure $2 \mathrm{a}$ shows the ESI mass spectrum, and Figure $2 b$ the transformed ESI mass spectrum of that fraction. Trypsin treatment of this mixture, when analyzed by LSIMS, showed the presence of inns at $m / z \quad 5.58$ (I) and 1134 (II) (among others) (Figure 3); those two ions correspond to $\mathrm{BE}_{20-24}\left(=\mathrm{POMC}_{254-258}\right)$ and $\mathrm{BE}_{10-19}\left(=\mathrm{POMC}_{244-253}\right)$, respectively [9]. Those two peaks of $m / z 558$ and 1134 were also present in the fast-atom bombardment (FAB) spectrum from the trypsin treatment of endogenous BE (M.W. 3465) in
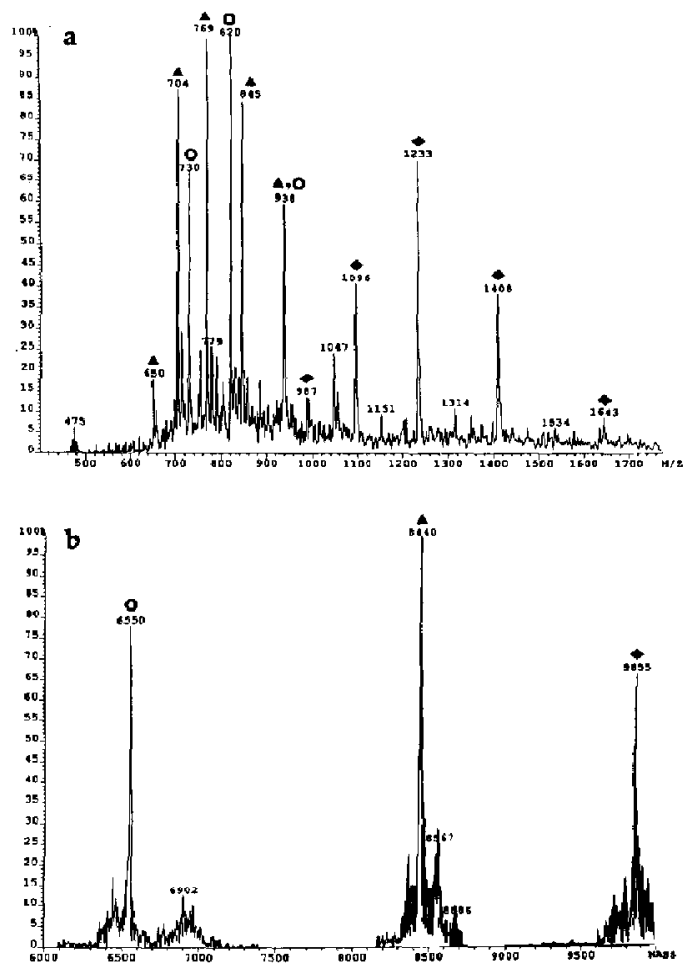

Figure 2. ESI mass spectrum of a fraction extracted from bovine pituitaries that contained ME-li. (a) ESI mass spectrum; (b) transformed ESI mass spectrum. Ions associated with $9855(\diamond), 8440$ $(4)$, and $6550(O)$ are indicated

our previous studies [6]. Therefore, those LSIMS data indicate that this fraction contains a $\mathrm{BE}$-containing protein.

That fraction was subjected to further IPLC separation and the component with a M.W. of 6550 was removed (data not shown). The component with a M.W. of 8440 was difficult to remove without a concomitant and unavoidable loss of the component with a M.W. of 9855 . The component with a M.W. of 8440 is not trypsin-sensitive because it remains in the ESI spectrum following trypsin treatment but 9855 disappears.

Therefore, the component with a M.W. of 9855 is the putative intermediate-sized precursor to $B E$ in bovine pituitary. More pituitary sample is being accumulated; that fraction will be subjected to further HPLC separation, and that putative BF-rontaining protein will be characterized by trypsin treatment, ESIMS, LSIMS, and MS/MS sequencing of each tryptic fragment. In that manner, that BE-containing protein with a M.W. of 9855 will be located within POMC. That result will permit the elucidation of the metabolic processing of POMC. 


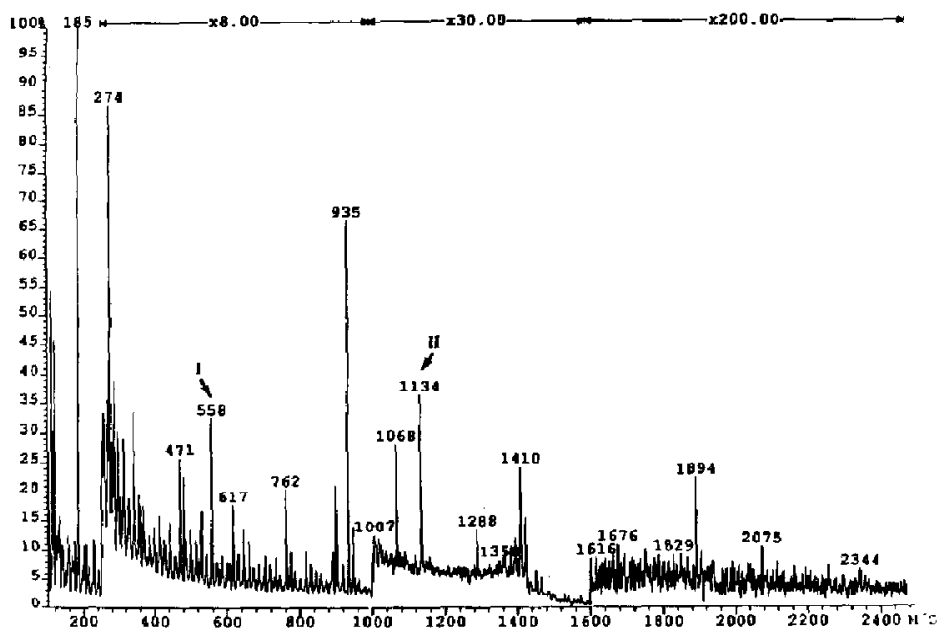

Figure 3. LSIMS spectrum of the mixture of peptides produced by trypsin treatment of the fraction that contained ME-li. Note the ions at $m / z 558$ (I) and 1134 (I), which correspond to $\mathrm{BE}_{20-24}$ and $\mathrm{BE}_{10-19,}$ respectively.

\section{Bovine $\alpha$-Lactalbumin}

Scheme III contains the experimental protocol used for the study of BAL. Three scparate experiments were conducted: I, M.W. determination; II, trypsin, no HPLC; and III, trypsin, with HPLC. In experiment I, BAL was analyzed directly by ESIMS to determine the M.W. of the protein, and again after DTT reduction to provide the M.W. of reduced BAL. The number of disulfide bonds in BAL was estimated by the difference in those two M.W. values.

In experiment II, BAL was treated with trypsin, and the mixture of tryptic peptides was screened by mass spectrometry. ESIMS and LSIMS provided the M.W. of the majority of the tryptic peptides. Some of the more abundant tryptic peptides in the mixture were analyzed further by LSIMS-MS/MS to obtain the amino acid sequence of the selected tryptic peptide. The mix-

I. BAL

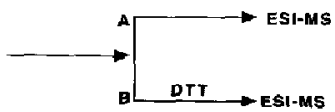

II. BAL
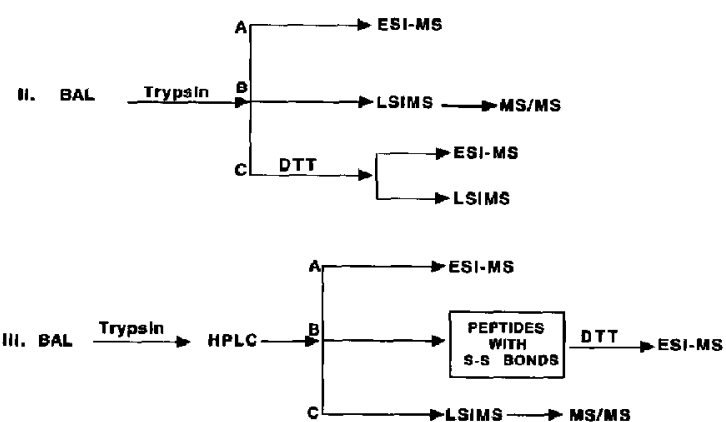

Scheme III. Experimental protocol used to analyze BAL by ESIMS, DTT reduction of disulfide bonds, trypsin treatment, LSIMS, MS /MS, and RP-HPLC. ture of the DTT-reduced tryptic peptides was analyzed by ESIMS and LSIMS to detect those peptides that contain disulfide bonds.

In experiment III, the mixture of tryptic peptides was separated by RP-HPLC, which is used when a sufficient amount of a biological sample is available. ESIMS and LSIMS-MS/MS analyzed the individual RP-HPLC-purified peptides. ESIMS, following DTT reduction, was used to detect those peptides that contain disulfide bonds.

Determination of the molecular weight, and the number of protonation sites, and an estimation of the number of disulfide bonds in bovine $\alpha$-lactalbumin. Molecular weight of BAL. Figure 4 a contains the ESI mass spectrum of BAL and Figure $4 \mathrm{~b}$ the corresponding mass spectrum after DTT reduction. The insets in Figure $4 a$ and $b$ contain the corresponding transformed ESIMS data. In Figure 4a, the observed ion species range from $7^{+}$to $12^{+}$, and in Figure $4 \mathrm{~b}$, from $8^{+}$to $19^{+}$. The M.W. of BAL is determined to be $14,174 \pm 2 \mathrm{Da}(n=5)$; the calculated average M.W. is 14,175 . The relative standard deviation of the five determinations is $\pm 0.02 \%$.

Number of protonation sites. In BAL, the number of basic amino acid residues plus the N-terminus of the protein is 17. From Figure $4 a$, the maximum number of charges observed in the ESI spectrum is 12. As mentioned by other researchers [20], during ESIMS several basic amino acids may remain buried within the structure of a folded protein such as BAL, and thus they would not be protonated. The folded structure is maintained by the four disulfide bonds in BAL.

It is interesting to speculate on which one of the basic amino acids in BAL remains buried and thus resistant to protonation in the disulfide-folded structure. The ${ }^{62}$ Lys residue (see Figure 1a) may be a candidate for such a buried residue because when trypsin (powder or beads) was used, that residue, which is located next to a disulfide bond, is incom- 

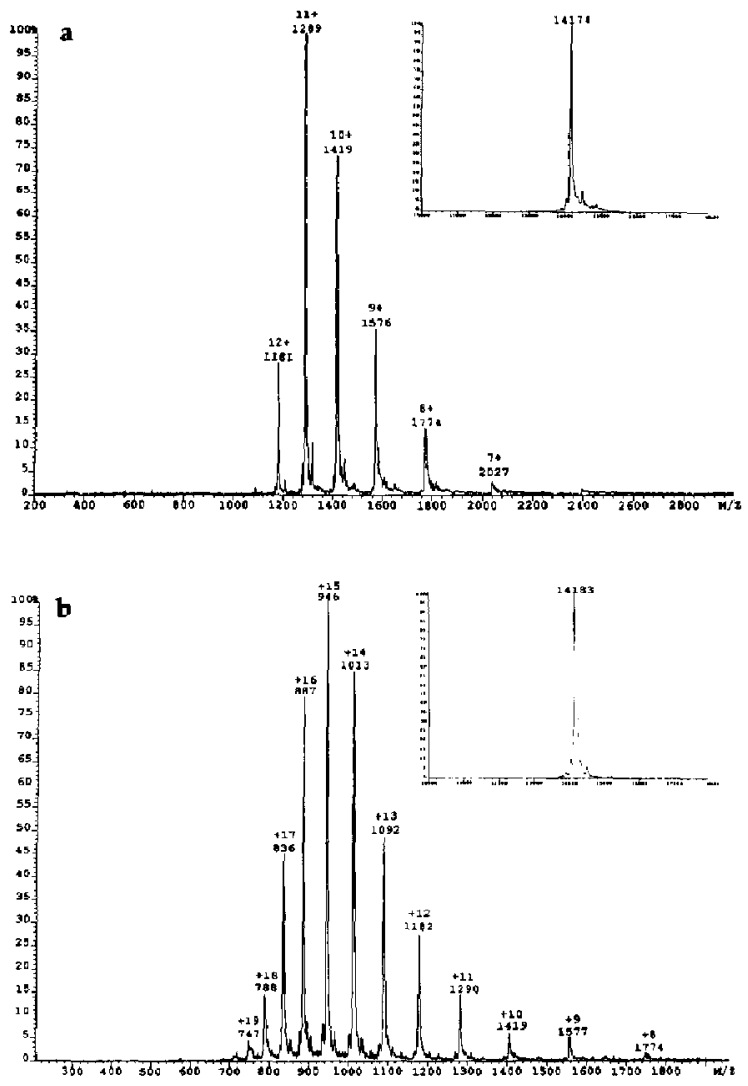

Figure 4. ESI mass spectrum of bovine $\alpha$-lactalbumin before (a) and after (b) DTT reduction of disulfide bonds. The insets in each mass spectrum show the corresponding transformed ESIMS data.

pletely hydrolyzed at its C-terminal bond (data not shown).

DTT can be used to reduce the disulfide bonds in a protein and open the folded structure into a more linear structure, thus allowing the buried basic amino acids to become available for protonation. After DTT reduction (see Figure $4 \mathrm{~b}$ ), the ESIMS spectrum shows that the maximum number of charges increased by 7 (from 12 to 19). That latter value approximates the number of amino acids that could be protonated in $\mathrm{BAL}(\mathrm{K}=12, \mathrm{R}=1, \mathrm{H}=3$, plus the amino terminus). Protonation of the Gln side chains in BAL is also a possibility [20]. Clearly, all of the basic amino acid residues of BAL have been protonated following DTT reduction of disulfide bonds using this present experimental protocol. Other researchers [20] have noted a mass shift following reduction, but the data in Figure $4 \mathrm{~b}$ show a higher level of disulfide bond reduction ( $\mathrm{a}$ shift towards $19^{+}$compared to the $16^{+}$, and a different intensity distribution, compared to the spectrum in Figure 4 in Ref. [20]) because nitrogen was used here during the reduction to minimize the reoxidation of DTT-reduced disulfide bonds.
Estimation of the number of disulfide bonds in BAL. The M.W. of DTT-reduced BAL is determined to be $14,183 \pm 2 \mathrm{Da}(\mathrm{n}=5)$; the RSD is $\pm 0.01 \%$. The M.W. of BAL increases by nine mass units following DTT reduction (Figure $4 \mathrm{~b}$ versus $4 \mathrm{a}$ ). That increase in mass correlates to the total number of disulfide bonds in BAL $(9 / 2=4.5$; truncate to $4 \pm 2)$. Similar data (not presented here) were obtained for the corresponding ESIMS analysis (with and without DTT) of lysozyme.

However, that difference in mass (Figure $4 \mathrm{a}$ versus $4 b$, transformed data) must be interpreted carefully. For example, for an unknown protein that contains disulfide bonds, several experimental parameters must be optimized to more accurately calculate the number of disulfide bonds: instrumental resolution, mass calibration, maximum amount of sample to provide an optimal number of scans, and optimal number of $[\mathrm{M}+\mathrm{nH}]^{\mathrm{n}+}$ peaks in an ESI mass spectrum.

During a study of opioid peptide-containing proteins in the bovine pituitary [15], this method was used to determine whether chromatographic fractions contained proteins with disulfide bonds (no disulfide bonds were found).

Analysis of tryptic peptides. No RP-HPLC separation. Figure $1 \mathrm{~b}$ contains a list of all the tryptic peptides that could be produced by trypsin treatment of $\mathrm{BAL}$, their amino acid sequence, and their M.W. as monoisotopic mass values. The mixture of tryptic peptides was analyzed directly by LSIMS and ESI without any HPLC separation, and those data are collected in Table 1 . This type of experiment on a mixture of tryptic peptides is useful as a very effective initial screen during some experiments (see Figure 3).

Figure 5 (the LSIMS spectrum of the mixture), contains $[\mathrm{M}+\mathrm{H}]^{+}$ions that correspond to the tryptic peptides with M.W. values of 374,388 , 487, 617,1200 , and 1684 (very weak).

Similarly, the ESI mass spectrum shown in Figure 6 of the mixture of tryptic peptides contains $[\mathrm{M}+\mathrm{nH}]^{\mathrm{n}+}$

Table 1. Peptides produced by trypsin treatment of BAL and analyzed with LSIMS and ESI (no DTT reduction); with and without RP-HPLC separation

\begin{tabular}{rrrr}
\hline & \multicolumn{2}{c}{ No HPLC } & $\frac{\text { HPLC }}{\text { M.W. }}$ \\
\cline { 2 - 4 } & LSIMS & ESI & ESI \\
\hline 374 & + & + & $(+)$ \\
388 & + & + & +1 \\
487 & + & + & + \\
617 & + & + & + \\
1200 & + & + & + \\
1684 & + & + & + \\
4071 & - & + & + \\
5301 & - & - & + \\
\hline
\end{tabular}

Plus = found; minus = not found; all masses are monoisotopic. rounded off to the nearest integer. (H) See text. The appropriate protonated molecule ions of these two peptides were found in the mixture of unresolved components that eluted within the HPLC solvent front. 


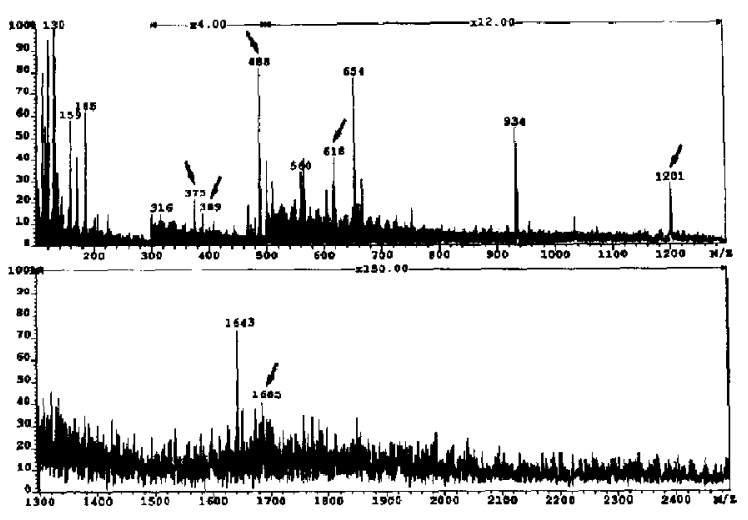

Figure 5. LSIMS spectrum of the mixture of peptides produced by trypsin treatment of bovine $\alpha$-lactalbumin. Pertinent peptides discussed in the text are indicated by arrows in this spectrum, and are listed in Table 1.

ions that correspond to the tryptic peptides with M.W. values of $374[375(1+)], 388[389(1+)], 487[488(1+)$, $244(2+)], 617$ [309 $(2+)$, very small, unlabeled], 1200 [601 $(2+)], 1684[843(2+), 562(3+)]$, and 4071 [1019 $(4+), 815(5+)]$. It is interesting to note that the largest disulfide-containing tryptic peptide with a M.W. of 5301 is missing in the ESI mass spectrum of the mixture contained in Figure 6. It is possible that ESI is not an effective mode for the analysis of this particular peptide in this mixture because of the small number (4) of charge sites compared to its large number (48) of amino acids.

The LSIMS product-ion spectra of selected tryptic peptides in the mixture produced by trypsin treatment of BAL (Scheme III, experiment IIB) were obtained. For example, note in Figure 5 the abundant $[\mathrm{M}+\mathrm{H}]^{+}$ ion at $m / z$ 488. Figure 7 contains the corresponding

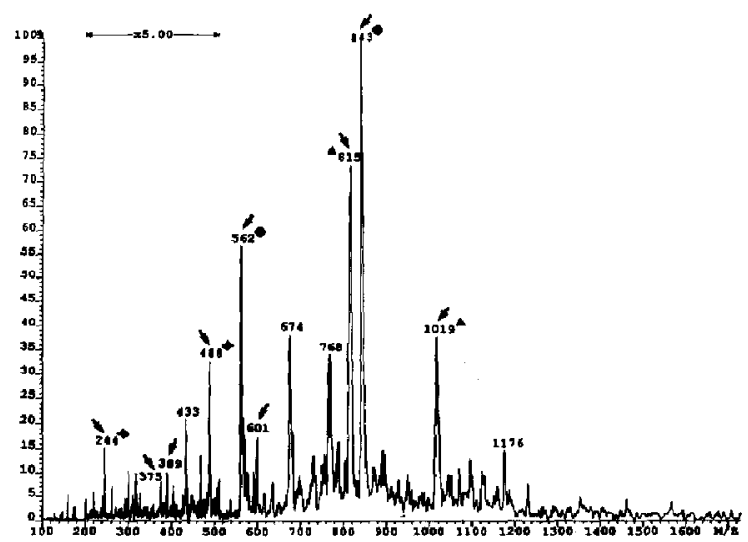

Figure 6. ESI mass spectrum of the mixture of peptides produced by trypsin treatment of bovine $\alpha$-lactalbumin. Pertinent peptides discussed in the text are indicated by arrows in this spectrum, and are listed in Table $1 ;(\Delta)$ ions associated with the peptide with a $M$. W. of 4071 , ( $(1684$, and $(\uparrow 487$.

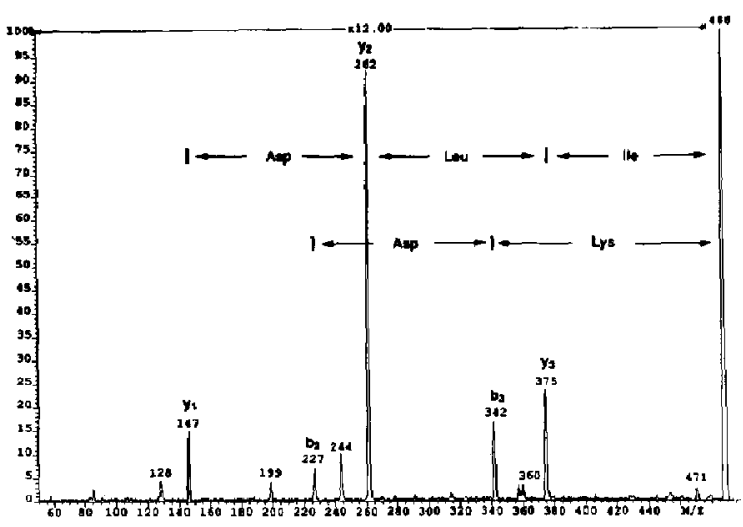

Figure 7. LSIMS-tandem mass spectrum of the precursor ion $m / z$ 488. That precursor ion is also shown in the LSTMS spertnum of tryptic peptides in Figure 5. Amino acid sequence-determining ions are indicated.

product-ion spectrum of that 488 ion, and contains amino acid sequence-determining fragment ions at $m / z 375$ ( $y_{3}$, loss of The/Leu), 262 ( $y_{2}$, loss of Leu/lle), and $147\left(\mathrm{y}_{1}\right.$, loss of Asp). Also, that 488 ion is the precursor for $342\left(b_{3}\right.$, loss of Lys) and $227\left(b_{2}\right.$, loss of Asp).

Two other precursor ions $(\mathrm{m} / z 375$ and 389) shown in Figure 5 were analyzed in that mixture by MS/MS, and excellent product-ion spectra were obtained (data not shown). The $[\mathrm{M}+\mathrm{H}]^{+}$ion at $m / z 375$ is the precursor for $260\left(\mathrm{y}_{2}\right.$, loss of Asp), $147\left(\mathrm{y}_{1}\right.$, loss of Leu/lle), and $229\left(b_{2}\right.$, loss of Lys). The other $[M+H]^{+}$ ion at $m / z 389$ is the precursor for 260 ( $\mathrm{y}_{2}$, loss of Glu) and 147 ( $y_{1}$, loss of Leu/Tle).

DTT reduction of disultide bonds of the tryptic peptides was performed (Scheme MI, Experiment IIC), and the corresponding LSIMS and ESI mass spectra are shown in Figures 8 and 9, respectively. As demonstrated by the data in Figure 1b, the disulfide-bond

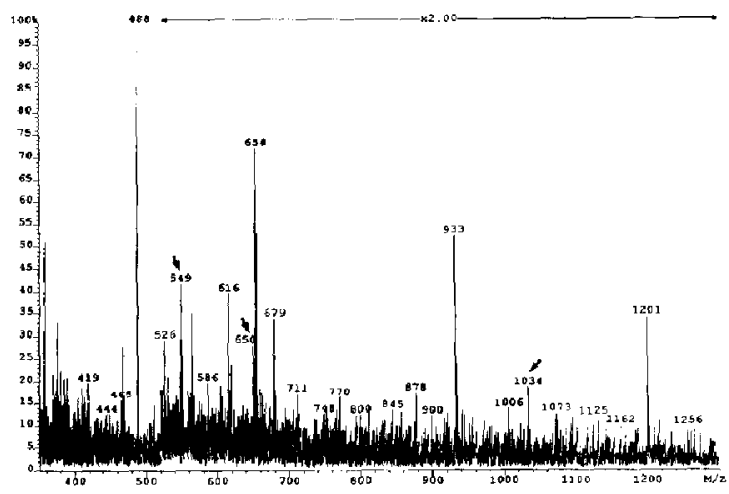

Figure 8. LSIMS spectrum of the mixture of peptides produced by trypsin treatment of bovine a-lactalbumin, followed by DTT reduction. Pertinent peptides discussed in the text are indicated by arrows and are listed in Table 2. 


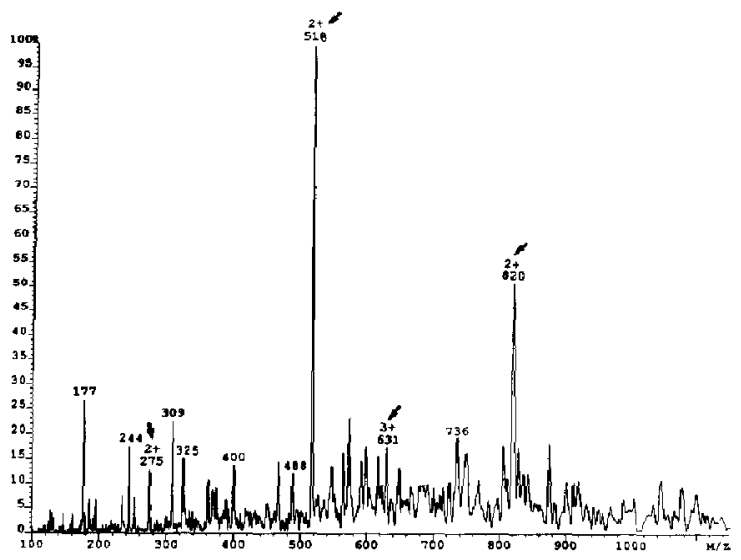

Figure 9. ESI mass spectrum of the mixture of peptides produced by trypsin treatment of bovine $\alpha$-lactalbumin, followed by DTT reduction. Pertinent peptides discussed in the text are indicated by arrows, and are listed in Table 2.

containing tryptic peptides with M.W. values of 1684 , 4071 , and 5301 are pertinent. The peptide at 1684 after DTT reduction produces two peptides with M.W. values of 652 and 1033 (see Table 2); 4071 produces three peptides with M.W. values of 548, 1638, and 1889; and 5301 produces two peptides with M.W. values of 649 and 4654. In the LSIMS spectrum (Figure 8), the ion at $m / z 1034$ corresponds to the peptide of M.W. 1033 in Table 2 and indicates the presence of the peptide with a M.W. of 1684; $m / z 650$ indicates 5301; and $m / z 549$ indicates 4071 .

Similarly, the ESI mass spectra (Figure 9) of that mixture indicates a peptide with a M.W. of 1684 by the peptides with ions at 1033 [518 (2+)]; 5301 by 4654 [1551 $(3+)$ in another ESI mass spectrum, not shown] and $649[325(2+)]$; and 4071 by $1638[820(2+)], 1889$ $[631(3+)]$, and $548[275(2+)]$.

RP-HPLC separation. Mass spectrometry data following RP-HPLC separation were obtained for the set of peptides produced by trypsin treatment of BAL (Scheme III, experiment III). Selected specific examples

Table 2. Peptides produced by trypsin treatment of BAL and analyzed after DTT reduction with LSIMS and ESI (with and withuut RP-HPLC separation)

\begin{tabular}{crrrrr}
\multicolumn{2}{c}{ Reduction } & & \multicolumn{2}{c}{ No HPLC } & HPLC \\
\cline { 4 - 6 } Before & After & & LSIMS & ESI & ESI \\
\hline \multirow{2}{*}{1684} & 652 & - & - & + \\
& 1033 & + & + & + \\
4071 & 548 & + & + & + \\
& 1638 & - & + & + \\
& 1889 & - & + & + \\
5301 & 649 & + & + & + \\
& 4654 & - & + & -
\end{tabular}

Plus $=$ found; minus $=$ not found: all masses are monoisotopic rounded off to the nearest integer.
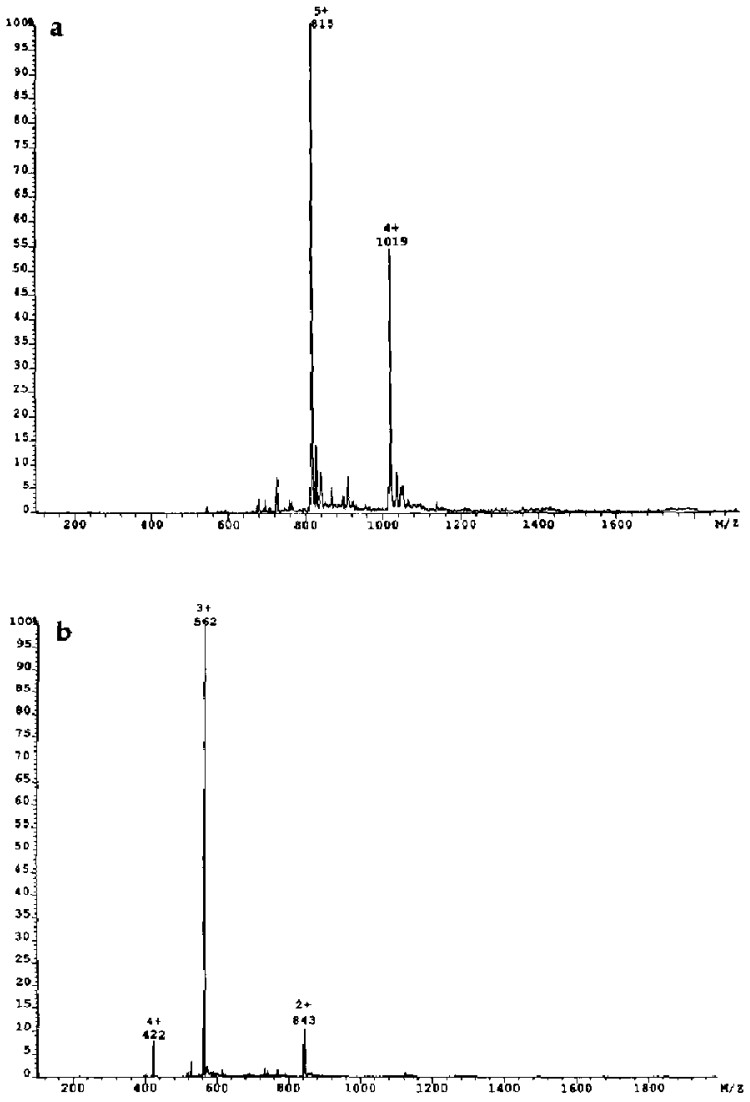

Figure 10. ESI mass spectra of HPLC-separated peptides produced by trypsin treatment of bovine $\alpha$-lactalbumin. (a) Peptide with a M. W. of $4071[1019(4+), 815(5+)]$; (b) Peptide with a M. W. of $1684[843(2+), 562(3+), 422(4+)]$.

will demonstrate the data listed in the Table $1 \mathrm{HPLC}$ column. ESI mass spectra were obtained for the separated peptides with M.W. values of $487,617,1200$, 1684,4071 , and 5301. (The peptides with M.W. values of 374 and 388 were eluted unresolved in the HPLC solvent front. The ESI mass spectrum (not shown) of that solvent front contained a mixture, in which $375^{+}$ and $389^{+}$were observed.) In addition, an ESI mass spectrum (not shown) was obtained for a peptide with a M.W. of 1797, which corresponds to the peptide at 1684 plus Leu; that peptide derives from an incomplete trypsinolysis of the C-terminal ${ }^{122} \mathrm{~K}-{ }^{123} \mathrm{~L}$ bond (see Figure 1a).

Figure 10a contains the ESI mass spectrum of the tryptic peptide with a M.W. of 4071, and shows the presence of ions at $m / z \quad 1019(4+)$ and $815(5+)$. Similarly, Figure $10 \mathrm{~b}$ contains the data for the peptide with a M.W. of 1684 , and shows ions at $m / z 843(2+)$, $562(3+)$, and $422(4+)$.

In Scheme III, experiment IIIB, BAL was treated with trypsin, and the mixture of tryptic peptides was 


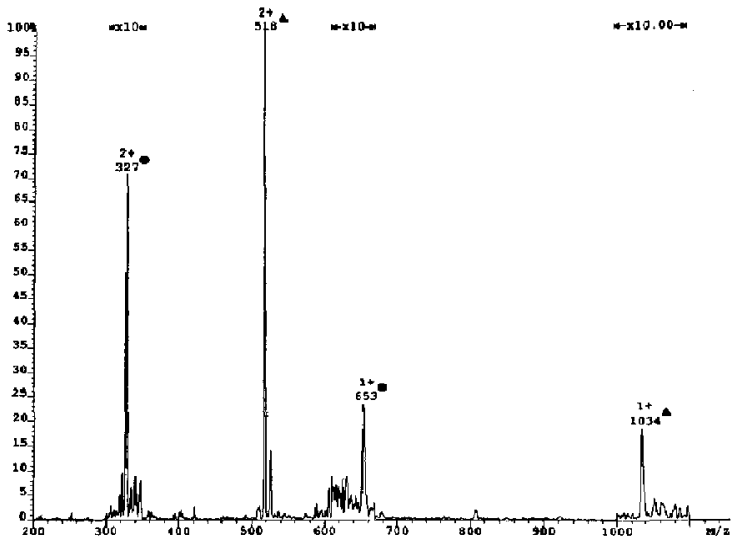

Figure 11. ESI mass spectrum of the HPLC-separated tryptic peptide with a M. W. of 1684 , followed by Li'T' treatment to produce peptides with $M$. W. values of $652(0), 653(1+), 327$ $(2+)$; and $1033(4) 1034(1+), 518(2+)$.

separated by RP-HPLC. Those tryptic peptides that contain disulfide bonds were treated with DTT and analyzed by ESIMS. As shown by the fragments listed in Figure $1 \mathrm{~b}$ and indicated in Table 2 by the products expected after DT" reduction, peptides with M.W. values of 652 and 1033 should be produced from the 1684 peptide. The ESI mass spectrum shown in Figure 11 contains ions that correspond to a peptide with a M.W. of $1033[1034(1+), 518(2+)]$, and the ions [653 $(1+), 327(2+)]$ indicate the presence of the peptide with a M.W. of 652 . These two DTT-reduced peptides $(652,1033)$ indicate the peptide with a M.W. of 1684. The data in Figure 12 are the corresponding data for the tryptic peptide with a M.W. of 4071, which had been reduced by DTT to produce three peptides with M.W. values of 548,1638 , and 1889 . The presence of the peptide with a M.W. of 548 is substantiated by the peaks at $m / z 549(1+)$ and $275(2+)$, the peptide at 1889 by $m / z 946(2+)$ and $631(3+)$, and the peptide at 1638 by $m / z 820(2+)$.

The tryptic peptide with a M.W. of 5301, following DTT treatment, is substantiated by the peak at $\mathrm{m} / \mathrm{z}$ $649[325(2+)]$ (dala nol shown).

Finally, data from experiment HIC are shown in Figure 13, which contains the LSIMS-tandem mass spectrum of the HPLC-purified tryptic peptide with a M.W. of 617 (see Table 1). Amino acid sequencedetermining fragment ions in this product-ion spectrum are seen at $m / z 489\left(\mathrm{y}_{4}\right.$, loss of Glu), 361 ( $\mathrm{y}_{3}$, loss of Gln), $248\left(\mathrm{y}_{2}\right.$, loss of Leu/Ile), and $147\left(\mathrm{y}_{1}\right.$, loss of Thr), and ions from the b-series at $258\left(b_{2}\right.$, loss of Thr-Lys) and $371\left(b_{3}\right.$, loss of Leu-Thr-Lys).

Interestingly, many peptides are found without any RP-HPLC separation by either ESIMS or LSIMS (Table 1); only the very large tryptic peptide with a M.W. of 5301 was not found without RP-HPLC separation (see discussion above). However, all tryptic peptides, including the peptide with a M.W. of 5301, were found

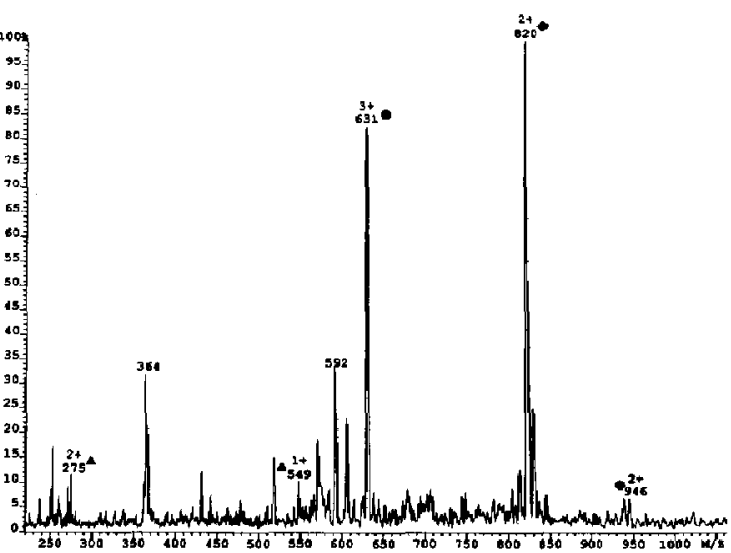

Figure 12. ESI mass spectrum of the HPLC-separated tryptic peptide with a M. W. of 4071 , followed by DTT treatment to produce peptides with $M . W$. values of $1638(\downarrow) 820(2+) ; 1889$ (C) $946(2+), 631(3+)$; and $548(4) 549(1+), 275(2+)]$.

when HPLC separation preceded mass spectrometry analysis. In general, ESIMS provided more data than LSIMS. Thus, trypsinolysis works fine on BAL before any disulfide bond reduction with DTT.

\section{Conclusions}

A BE-containing protein extracted from bovine pituitary and a commercially available protein can be characterized by a combination of mass spectrometry methods. For example, ESIMS indicates that the M.W. of the BE-containing protein is $9855 \mathrm{Da}$. Further, LSIMS of the mixture of peptides produced by trypsin treatment of that protein shows the presence of two unique tryptic peptides (M.W. values of 557 and 1133). The amino acid sequence of those tryptic peptides, and

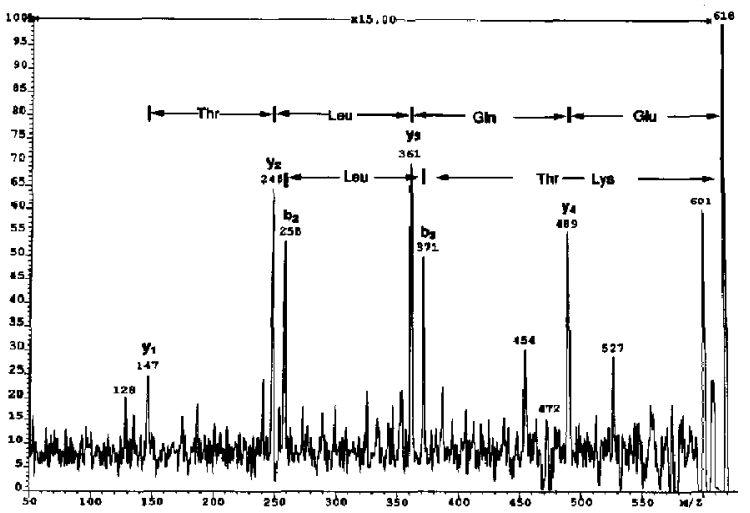

Figure 13. LSIMS-tandem mass spectrum of the HPLC-purified tryptic peptide with a $\mathrm{M}, \mathrm{W}$. of 617 . Amino acid sequencedetermining fragment ions of the $y$-series and b-series are indicated. 
thus of the 9855 protein, is currently being determined, and those data will locate that BE-containing protein within POMC.

BAL has served well as a model protein for these studies. BAL has been analyzed by ESIMS, DTT reduction of disulfide bonds, trypsin treatment, RP-HPLC separation, LSIMS, and MS/MS. This study was performed because a model compound was required for the method development for the separation and analysis of opioid peptide-containing proteins obtained from the pituitary (Refs. [14, 15]; Fridland, G. and Desiderio, D. M., Analyt. Lett., submitted).

ESIMS was used to determine the M.W. of HPLCpurified BAL, and trypsin treatment to produce a mixture of peptides that is analyzed (with and without RP-HPLC) by ESIMS, LSIMS, and MS/MS of selected precursor ions in the LSIMS spectrum of the mixture of tryptic peptides. DTT was used to reduce disulfide bonds in BAL and the tryptic peptides.

This study illustrated several important experimental features that are applicable to the analysis of proteins in general, and to opioid peptide-containing proteins and BAI, in particular: one peptide bond $\left({ }^{62} K^{-63} \mathrm{~N}\right)$ in BAL is relatively difficult to hydrolyze with trypsin; DTT reduction of disulfide bonds is important to uncover hidden basic amino acid residues; and it is important to avoid any reoxidation of a DTT-reduced protein.

The study of BAL is important for analyzing opioid peptide-containing proteins. The characterization of each opioid peptide-containing protein and their placement within POMC will be used to clarify the metabolic processing of that opioid neuropeptidergic system. Then, a comparison of that processing will be made between control pituitaries and postsurgical pituitaries (especially neuroendocrinologically silent tumors) to help clarify those mechanisms that contribute to tumor formation in the human anterior pituitary.

\section{Acknowledgments}

The authors gratefully acknowledge the financial assistance of the National Institutes of Health (GM 26666 to DMD), and the typing assistance of Virginia K. Scura and Vicki Antwine.

\section{References}

1. Desiderio, D. M. In Mass Spectrometry: Clinical and Biomedical Applications, Vol. 1; Desiderio, D. M. Ed.; Plenum Press: New York, NY, 1992; pp. 133-165.

2. Kaneko, T.; Wood, G.; Crouch, W. L.; Desiderio, D. M. Anal. Biachem. 1993, 215, 45-53.

3. Kaneko, T.; Wood, G.; Crouch, W. L.; Desiderio, D. M. Peptides, 1994, 15, 41-47.

4. Zhu, X.; Desiderio, D. M. J. Chromalogr. Biomed. Appl. 1993, $616,175-187$.

5. Kusmierz, J. J.; Sumrada, R.; Desiderio, D. M. Anal. Chem. 1990, 62, 2395-2400.

6. Kusmierz, J.; Dass, C.; Robertson, J. T.; Desiderio, D. M. Int. J. Mass Spectrom. Ion Processes 1991, 111, 247-262.

7. Desiderio, D. M.; Wyler, A. R.; Somes, G. J. Epilep. 1992, 5, $105-110$.

8. Desiderio, D. M.; Kusmierz, J. J.; Zhu, X.; Dass, C.; Hilton, D.; Robertson, J. T.; Sacks, H. S. Biol. Mass Spectrom. 1993, 22, $89-97$.

9. Dass, C.; Kusmierz, J. J.; Desiderio, D. M. Biol. Mass Spectrom. 1991, 20, 130-138.

10. Dass, C.; Kusmierz, J. J.; Desiderio, D. M.; Jarvis, S. A.; Green, B. N. J. Am. Soc. Mass Spectrom. 1991, 2, 149-156.

11. Lovelace, J. L.; Kusmierz, J. J.; Desiderio, D. M. J. Chromatogr. Biomed. Appl. 1991, 562, 573-584.

12. Tinsley, P. W.; Dass, C.; Trimble, J. W.; Desiderio, D. M. Exp. Eye Res. 1990, 51, 671-674.

13. Burgus, R.; Dunn, T.; Desiderio, D. M.; Ward, D. N.; Vale, W.; Guillemin R. Nature 1970, 226, 321-375.

14. Tanzer, F. S.; Tolun, E.; Fridland, C. H.; Dass, C.; Killmar, J.; Tinsley, P. W.; Desiderio, D. M. Int. J. Pept. Prot. Res. 1988, 32, $117-122$.

15. Yan, L.; Fridland, G.; Tseng, J.; Desiderio, D. M. Biochem. Biophys. Res. Commun. 1993, 196, 521-526.

16. Numa, S. In The Peptides: Analysis, Synthesis, Biology; Udenfriend, S.; Meienhofer, J., Eds. Academic Press: New York, 1984; pp. 1-23.

17. Dayhoff, M. O. Allas of Protein Sequence and Structure; National Biomedical Research Foundation: Washington, DC, 1972; p. 136.

18. Konigsbor, W. In Methads in Enzymology. Vol. XXV (Enzyme Struchure Part B); Hirs, C. H. W.; Timasheff, S. N., Eds.; 1972; pp. $185-188$.

19. Desiderio, D. M.; Cunningham, M. J. Liq. Chromatogr. 1981, 4, 721-733.

20. Loo, J. A.; Edmonds, C. G.; Usdeth, H. R.; Smith, R. D. Anal. Chem. 1990, 62, 693-698.

21. Smith, D. L.; Sun, Y. In Mass Spectrometry of Peptides; Desiderio, D. M. Ed.; CRC Press: Boca Raton, FL, 1991; pp. 275-280. 